Check for updates

Cite this: RSC Adv., 2017, 7, 37858

Received 19th May 2017

Accepted 25th July 2017

DOI: $10.1039 / c 7 r a 05621 d$

rsc.li/rsc-advances

\section{Ginkgolide B lowers body weight and ameliorates hepatic steatosis in high-fat diet-induced obese mice correlated with pregnane $\mathrm{X}$ receptor activation}

Lingling Luo, Yin Li, Dongshan Wang, Yuanyuan Zhao, Yahui Wang, Fei Li, Jinan Fang, Hui Chen, Shengjie Fan and Cheng Huang (D) *

Ginkgolide $B(G B)$ is a natural occurring terpene lactone and has pharmacological function in the cardiovascular system. In the screening of pregnane $X$ receptor (PXR) ligands, we found that GB is a PXR agonist. In the present study, we investigated the effects of GB on metabolic disorders in highfat (HF) diet-induced obesity (DIO) C57BL/6 mice. A reporter gene assay was used to analyze the effects of GB on human PXR ( $h P X R$ ) transactivity. DIO mice were divided into three groups randomly, and were fed with $\mathrm{HF}$ alone, or HF mixed with $0.1 \% \mathrm{~GB}(\mathrm{w} / \mathrm{w})$ or HF mixed with $0.025 \%$ obeticholic acid (OCA) for 7 weeks. Control lean group mice were fed with a normal diet (chow). Body weight was measured every other day. At the end of the treatment, the fasting blood glucose, glucose tolerance, serum lipid profile, liver lipid content and morphology of the liver of DIO mice were analyzed. The gene expression analysis was performed with a quantitative real-time PCR assay. The results showed that GB activated hPXR transactivity in a dose dependent manner, while GB did not change peroxisome proliferator-activated receptor (PPAR) $\alpha, P P A R \beta, P P A R \gamma$, liver $X$ receptor (LXR) $\alpha, L X R \beta$ and farnesyl $X$ receptor (FXR) transactivities. GB treatment reduced body weight and serum triglyceride (TG) levels, and ameliorated hepatic steatosis in DIO mice. A mechanistic study showed that GB increased the mRNA expression of PXR target genes in the DIO mouse liver. Our study suggests that GB may reduce body weight, lower serum TG and improve lipid accumulation in the liver of DIO mice through the activation of PXR signaling.

\section{Introduction}

Metabolic syndrome is a worldwide problem and its prevalence is increasing at a staggering pace. ${ }^{1}$ It is characterized by obesity, hypertension, dyslipidemia, hyperglycemia along with insulin resistance. $^{2}$ Nonalcoholic fatty liver disease (NAFLD) is also considered the hepatic manifestation of metabolic dysfunction, which makes up a spectrum ranging from NAFLD to liver fibrosis and cirrhosis. ${ }^{3}$ Currently there is no approved drug for NAFLD. Therefore, therapy for NAFLD is urgently needed.

Pregnane X receptor (PXR, NR1I2) is a ligand-activated nuclear hormone receptor expressed predominantly in the liver and small intestine. ${ }^{4}$ It was initially identified as a "xenobiotic sensor" that plays a central role in regulating the expression of genes involved in detoxification and bioactivation. ${ }^{5}$ Recently it has been reported that PXR activation could repress gluconeogenesis. ${ }^{6}$ In mice, the activation of PXR

Drug Discovery Lab, School of Pharmacy, Shanghai University of Traditional Chinese Medicine, Shanghai 201203, China. E-mail: chuang@shutcm.edu.cn; Fax: +86-2151322193; Tel: +86-21-51322182 by pregnenolone-16 $\alpha$-carbonitrile (PCN) decreased fasting blood level glucose and protected from weight gain under a high-fat diet. ${ }^{7}$ The PXR transgenic mouse study has revealed the involvement of PXR in hepatic lipogenesis and fatty acid oxidation. ${ }^{8}$ Clinical studies showed that PXR protein level was lower in severe non-alcoholic steatohepatitis (NASH) patients, ${ }^{9}$ suggesting that PXR is involved in regulating energy homeostasis, lipid and glucose metabolism, bile acid homeostasis and hepatic steatosis under various physiological and pathological conditions. Targeting PXR may be a strategy for therapy of metabolic diseases.

Ginkgolide B (GB) is a major terpene lactone in the roots and leaves of Ginkgo biloba.$^{10}$ It is a potent antagonist of plateletactivating factor (PAF) receptor. ${ }^{11}$ Previous studies have reported that GB may have therapeutic effects on ischemic brain injury, ${ }^{12}$ platelet aggregation and thrombosis ${ }^{13}$ inflammatory diseases,${ }^{14}$ tumor, ${ }^{15}$ migraine.${ }^{16}$ Notably, previous reports have reveals the multiple Ginkgo biloba extract on obese mice. ${ }^{17}$ In the present study, we identified that GB could improve the high-fat diet-induced metabolic disorders in DIO mice. 


\section{Materials and methods}

\subsection{Cell cultures and reporter assays}

The reporter assay was performed as previously described. ${ }^{18}$ Briefly, HEK 293T cells obtained from ATCC were maintained in DMEM supplemented with $10 \%$ fetal bovine serum (FBS, Hyclone, Logan, UT, USA). For cell plates, the expression plasmid PSG5-hPXR and CYP3A4-Luc, pCMXGal-hPPAR $\alpha, \beta, \gamma$ LBD, LXR $\alpha$, $\beta$ LBD, and the Gal4 reporter vector MH100×4-TK-Luc were cotransfected with pREP7 (Renilla luciferase). For transfection, each well contained $100 \mathrm{ng}$ of total plasmids and 0.2 $\mu$ of FuGENEHD transfection reagent (Roche, Germany). After $24 \mathrm{~h}$, PXR, PPAR $\alpha, \operatorname{PPAR} \beta, \operatorname{PPAR} \gamma, \mathrm{LXR} \alpha, \mathrm{LXR} \beta$, FXR agonist (Rifampicin, fenofibric acid, GW4064, Pioglitazone TO901317 and GW4064, respectively, Sigma Aldrich, St. Louis, MO, USA) and GB were added to fresh media and the cells were incubated for another $24 \mathrm{~h}$. The luciferase activities were measured using the DualLuciferase Reporter Assay System (Promega, USA), and the transfection efficiencies were normalized according to Renilla luciferase activity. All of the transfection experiments were performed in triplicate.

\subsection{Animals and diets}

The animal study protocols were approved by Shanghai University of Traditional Chinese Medicine (approval number: SZY201609004). Female C57BL/6 mice were purchased from SLAC Laboratory (Shanghai, China) at 7 weeks of age. All animals were kept under controlled temperature $22-23{ }^{\circ} \mathrm{C}$ on a $12 \mathrm{~h}$ light/ dark cycle. After a one-week adaptation period, the chow-control mice were fed a chow diet (10\% of calories derived from fat, D12450B, Research Diets, New Brunswick, NJ, USA). The rest of the mice were fed with a HF diet $(60 \%$ of calories derived from fat, D12492, Research Diets, New Brunswick, NJ, USA) for 3 months to induce obesity. Then the obese mice were randomly divided into three groups: HF group, OCA group (HF diet mixed with obeticholic acid at $0.025 \mathrm{~g} / 100 \mathrm{~g}$, Topharman Shanghai Co., Ltd), GB group (HF diet mixed with GB at $0.1 \mathrm{~g} / 100 \mathrm{~g}$, Chengdu
Push Bio-technology Co., Ltd). Mice were treated for 7 weeks. Food intake and body weight were measured every other day.

\subsection{Intraperitoneal glucose tolerance test}

At the end of treatment, mice were fasted overnight (12 h). Fasting glucose levels were determined from the tail vein $(0$ min) before the injection of glucose $\left(1 \mathrm{~g} \mathrm{~kg}^{-1}\right.$ body weight). Then blood samples were collected at regular intervals $(15,30$, 60, 90 and $120 \mathrm{~min}$ ) for glucose tolerance tests.

\subsection{Serum chemistry analysis}

The mice were fasted overnight and anesthetized (urethane, $20 \%, \mathrm{w} / \mathrm{v}$ ), and cardiac blood was taken. The blood samples were centrifuged at $800 \mathrm{~g}$ for $15 \mathrm{~min}$ to separate serum from the samples. Serum TG, total cholesterol (TC), high-density lipoprotein cholesterol (HDL-c), low-density lipoprotein cholesterol (LDL-c), total bile acid (TBA), alanine aminotransferase (ALT), aspartate transaminase (AST) levels were examined by an automatic analyzer (Hitachi 7020, Tokyo, Japan) using $100 \mathrm{ml}$ of heart blood serum.

\subsection{HE staining and oil red $O$ staining}

For H\&E staining, liver tissues were fixed in $4 \%$ paraformaldehyde, embedded in paraffin, cut into $5 \mu \mathrm{m}$ sections and stained with hematoxylin and eosin. For oil red O staining, frozen liver tissues were stained with oil red O (Sigma Aldrich, St. Louis, MO, USA).

\subsection{Fecal lipid content analysis}

Mice feces $(100 \mathrm{mg})$ were homogenized with $1 \mathrm{ml}$ of chloroformmethanol $(1: 1)$ and placed overnight. Then the homogenate was centrifuged (10 $\mathrm{min}$ at $900 \mathrm{~g})$. Supernatant was removed to new tubes, evaporated to dryness and dissolved in isopropanol to measure TG and TC levels with TG and TC assay kits according to the manufacturer's instructions (KINGHA WK, China).

Table 1 Sequence of the primers used in real-time PCR

\begin{tabular}{lll}
\hline Gene & Forward primer & Reverse primer \\
\hline$\beta$-Actin & TGTCCACCTTCCAGCAGATGT & AGCTCAGTAACAGTCCGCCTAGA \\
CYP3A11 & TCCAAATGATGTGCTGGTGAT & CTTGAGGCAGAAGGCAAAGAA \\
MRP2 & CAAGTCCAGATCATCGCTTCC & GCTTTCACCTCCATCACCCTC \\
MRP3 & CACCTTCCAGCTTCTCCATCT & AGCCTAAACATTCAAATCCCG \\
MDR1 & GAATGCTCCAATTAACACGGA & CTAAAAGGCTGGGGATAAAGA \\
CYP7A1 & GTGGTAGTGAGCTGTTGCATATGG & CACAGCCCAGGTATGGAATCA \\
SREBP1 & GGCTATTCCGTGAACATCTCCTA & ATCCAAGGGCAGTTCTTGTG \\
ACC & GAATCTCCTGGTGACAATGCTTATT & GGTCTTGCTGAGTTGGGTTAGCT \\
SCD-1 & TCACCTTGAGAGAAGAATTAGCA & TTCCCATTCCCTTCACTCTGA \\
FAS & CTGAGATCCCAGCACTTCTTGA & GCCTCCGAAGCCAAATGAG \\
CD36 & GCTTGCAACTGTCAGCACAT & GCCTTGCTGTAGCCAAGAAC \\
IL-1 $\beta$ & TCGTGCTGTCGGACCCATAT & GGTTCTCCTTGTACAAAGCTCATG \\
MCP-1 & AGGTCCCTGTCATGCTTC & GTGCTTGAGGTGGTTGTG \\
TNF- $\alpha$ & ATGGATCTCAAAGACAACCAACTAG & ACGGCAGAGAGGAGGTTGACTT \\
FGF15 & TGAAGACGATTGCCATCAAG & GAGTAGCGAATCAGCCCGTA
\end{tabular}




\subsection{Quantitative real-time PCR}

Total RNA was extracted from the livers and the small intestines of mice using Trizol reagent (TaKaRa, Shiga, Japan). The first strand cDNA was synthesized using a cDNA synthesis kit (Fermentas, Madison, WI, USA), and gene expression levels were analyzed by quantitative real-time RT-PCR using the ABI StepOnePlus Real Time PCR system (Applied Biosystems, Grand Island, NY, USA). Sequences for primers are listed in Table 1 . The mRNA levels of all genes were normalized using $\beta$-actin as an internal control.

\subsection{Hepatic glutathione content analysis}

Liver tissues were homogenized in $1 \mathrm{ml}$ saline and centrifuged at $8500 \mathrm{~g}$ for 10 minutes. The protein content of supernatants was determined using the bicinchoninic acid assay (Beyotime Biotechnology, Shanghai, China). Hepatic glutathione content was measured by a kit (Beyotime Biotechnology, Shanghai, China) according to manufacture's instruction.

\subsection{Statistical analysis}

All data were presented as means \pm SEM. Differences between the groups were examined for statistical significance using analysis of variance (ANOVA) and independent samples $t$-test (SPSS 21.0). $P<0.05$ was considered as statistically significant.
A

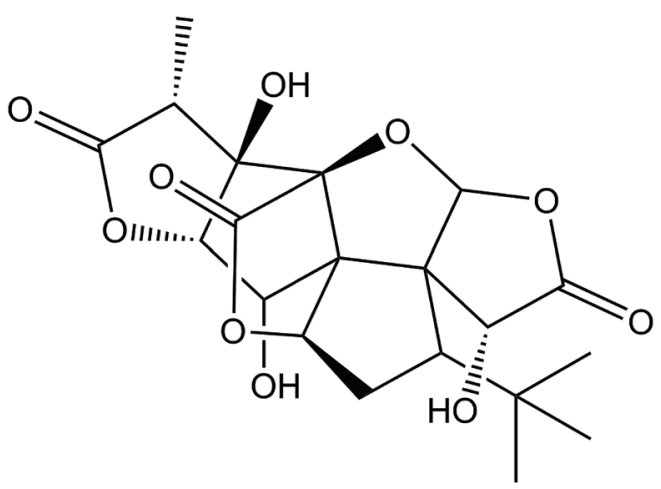

B

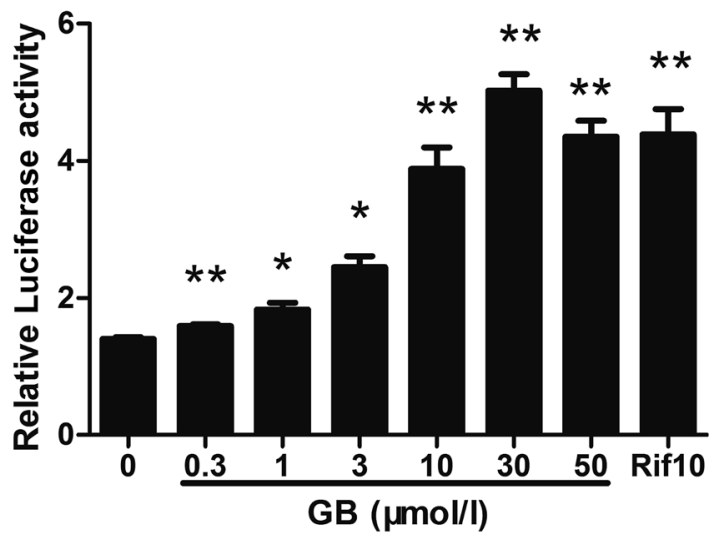

E

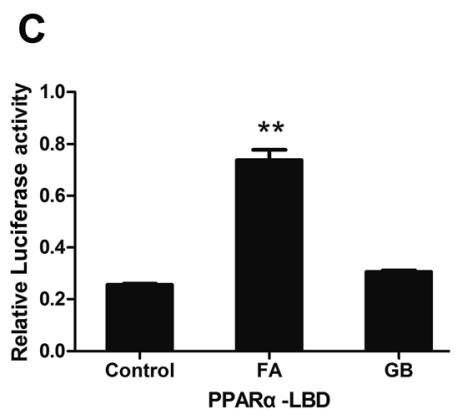

$\mathbf{F}$

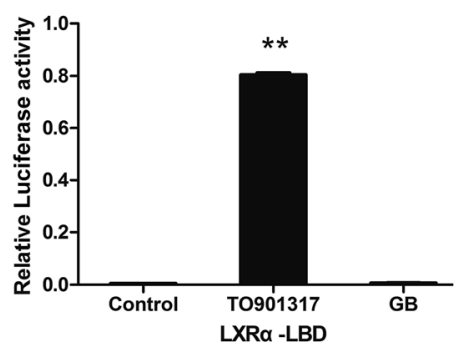

D

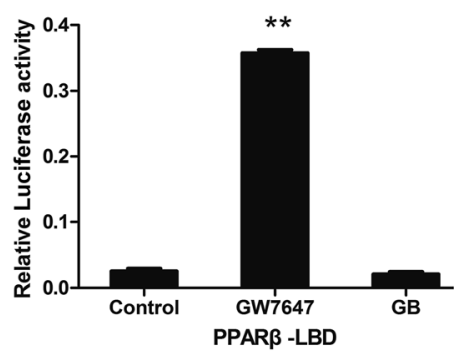

G

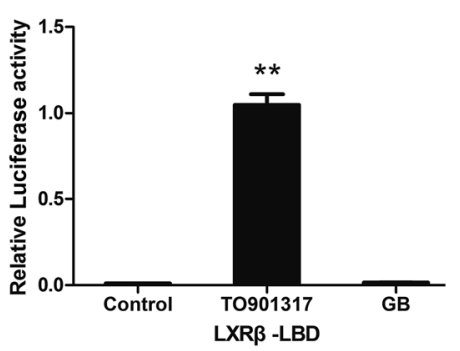

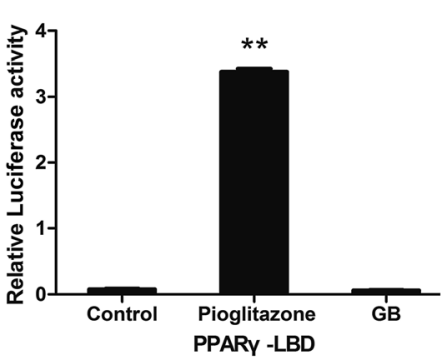

H

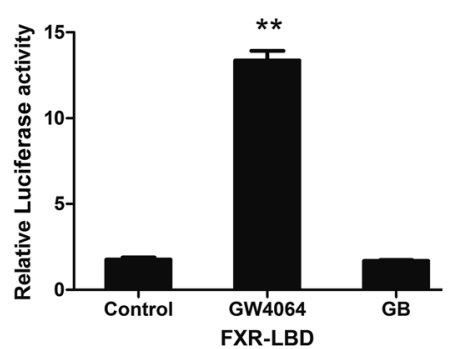

Fig. 1 Ginkgolide B activates the human PXR (hPXR). (A) Chemical structures of ginkgolide B (GB). (B) GB activates hPXR transactivity in a dosedependent manner. Full-length PSG5-hPXR was co-transfected with CYP3A4-Luc reporter construct to HEK 293T cells and treated with GB and Rif $10 \mu M$ for 24 h. (C-H) PPAR $\alpha$, PPAR $\beta$, PPAR $\gamma$, LXR $\alpha$, LXR $\beta$, FXR transactivity. HEK 293T cells were co-transfected with pCMXGal-hPPAR $\alpha, \beta, \gamma$ LBD, LXR $\alpha, \beta$ LBD, FXR LBD and the Gal4 reporter vector MH100 $\times 4$-TK-Luc expression plasmids for $24 \mathrm{~h}$ and treated with $10 \mu M$ PPAR $\alpha$, PPAR $\beta$, PPAR $\gamma, L X R \alpha, L X R \beta, F X R$ agonist (FA: fenofibric acid, GW7647, pioglitazone, TO901317 and GW4064). The relative luciferase activities were measured by comparison to Renilla luciferase activities. The results represent at least three independent experiments. The data are shown as means $\pm \operatorname{SEM}(n=3) . * P<0.05, * * P<0.01$ compared to the control. 


\section{Results}

\subsection{Ginkgolide B activates human PXR transactivity}

We used reporter assay to screen the ligands of hPXR from a 700 natural product library. The activity of hPXR was determined using a PXR-responsive CYP3A4-Luc reporter after drug treatment. ${ }^{19}$ The results showed that GB (Fig. 1A) induced transcriptional activity of hPXR in a dose-dependent manner (Fig. 1B), which was similar to hPXR specific agonist rifampicin (Rif). Meanwhile, we analysed the effects of GB on the transactivity of other important nuclear receptors that involved in the glucose and lipid metabolism showed that there were no effects of GB on PPAR $\alpha, \operatorname{PPAR} \beta, \operatorname{PPAR} \gamma, \operatorname{LXR} \alpha, \operatorname{LXR} \beta$ and FXR transactivities (Fig. 1C-H), indicating that GB may be a selective agonistic ligand of hPXR.

\subsection{Ginkgolide B prevents diet-induced obesity in C57BL/6 mice}

Since PXR is involved in glucose and lipid metabolism, we investigated whether GB could improve metabolic disorders in high-fat diet induced obese mice. As a result, the obese mice displayed a higher body weight gain than the chow diet mice. GB supplementation obviously blocked the body weight gain in the obese mice (Fig. 2A). The food intake amounts were no difference between the HF, GB and OCA groups (Fig. 2B), indicating that the body weight loss was not caused by the less food intake. Next, we tested fecal TG and TC contents. The GB group had a higher TG level in feces than the HF group, but not TC (Fig. 2C and D), suggesting GB may suppress the absorption of TG in intestine. In particular, we examined the weight of abdominal visceral white adipocyte tissues (WAT). The result

A

B
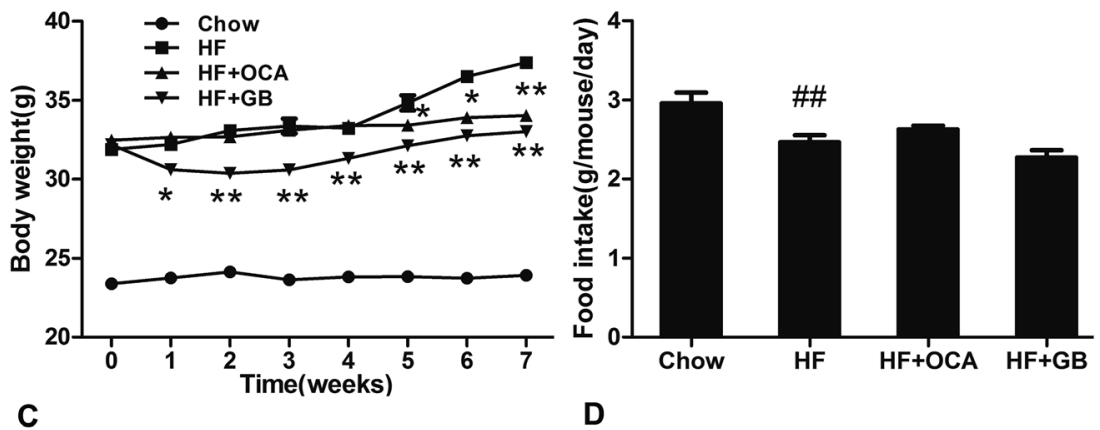

D

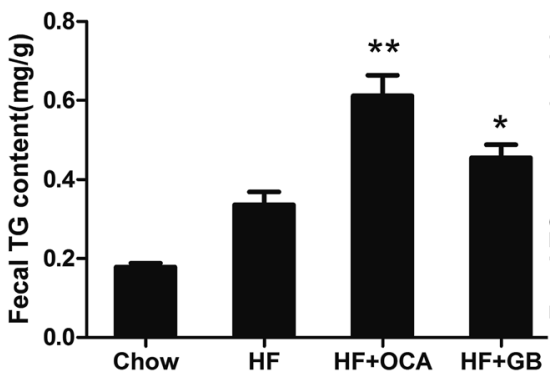

E

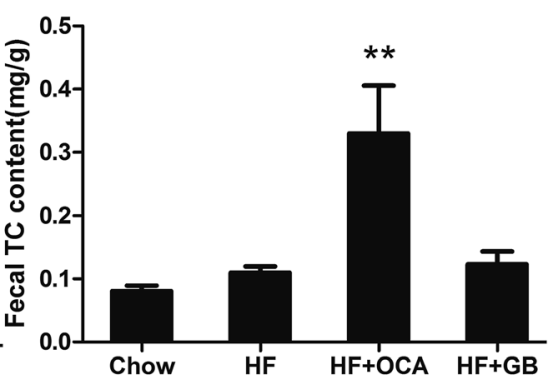

$\mathbf{F}$
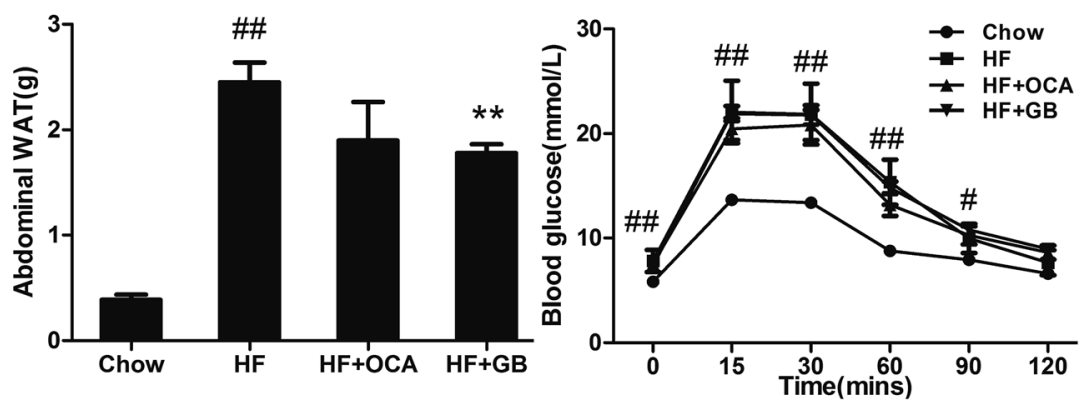

Fig. 2 Ginkgolide B prevents diet-induced obesity. (A) Body weight. (B) Food intake amount. (C) Fecal triglyceride levels. (D) Fecal total cholesterol levels. (E) The weight of abdominal visceral white adipocyte tissues (WAT). (F) Intraperitoneal glucose tolerance test (IPGTT). The mice were fasted overnight, and intraperitoneally injected with $1 \mathrm{~g}$ of glucose per kg body weight. The blood glucose levels were measured using tail vein blood at the times indicated and the basal glucose levels are shown at 0 min. The data are shown as means $\pm S E M(n=8)$. \#P<0.05, \#\#P< 0.01 , compared to the chow group; $* P<0.05, * * P<0.01$ compared to the HF group. 
showed that WAT weight in GB treated mice was reduced in comparison with that of control DIO mice (Fig. 2E).

It has been reported that hyperlipidemia and insulin resistance are relevant to metabolic disorders. ${ }^{20}$ To reveal whether GB could improve insulin resistance, we measured the fasting blood glucose levels and glucose tolerance of the mice. As shown in Fig. 2F, the glucose levels in HF diet-fed mice were markedly higher than those in the chow diet-fed mice. While GB treatment displayed a tendency that the glucose levels were reduced without reaching significance when compared with HF diet-fed mice.

\subsection{Ginkgolide B improves the lipid profiles and reduces serum total bile acid}

As expected for the DIO mice, serum TC, TG, LDL-c and HDL-c levels had a marked rise (Fig. 3A-D). In contrast, the GB treated obese mice showed a significant decrease serum TG level (Fig. 3A). However, we did not observe difference in the serum TC, LDL-c and HDL-c levels between GB treated group and DIO control mice, though that displayed a tendency to reduction. Since OCA treatment effectively improves $\mathrm{NASH},{ }^{\mathbf{2 1}}$ the OCA treatment group was designed as positive control. Interestingly, the OCA treatment markedly reduced the serum TC, LDL-c and HDL-c, but not TG in DIO mice.

Bile acid is steroid acid involved in lipid and cholesterol metabolism. In view of the previous studies, an elevation of bile acid was observed on DIO mice. ${ }^{22,23}$ To investigate the effect of GB on bile acid metabolism, the serum totally bile acid (TBA) was analyzed. The OCA group reduced TBA in serum (Fig. 3E), which was consistent with early studies that OCA was an effective drug for the treatment of cholestasis. ${ }^{24}$ Similarly, our results shown TBA in the GB group was lower than that in the DIO control group, suggesting that GB may regulate bile acid metabolism (Fig. 3E).

\subsection{Ginkgolide B ameliorates hepatic steatosis}

Obese mice usually develop hepatic steatosis due to excess fatty storage in liver. ${ }^{25}$ In our study, HE stained liver sections showed

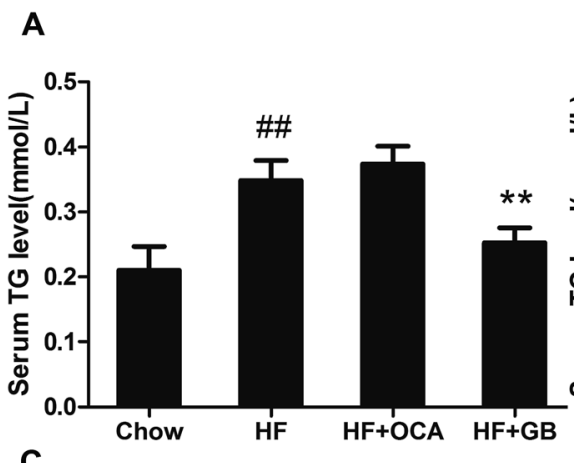

B

C
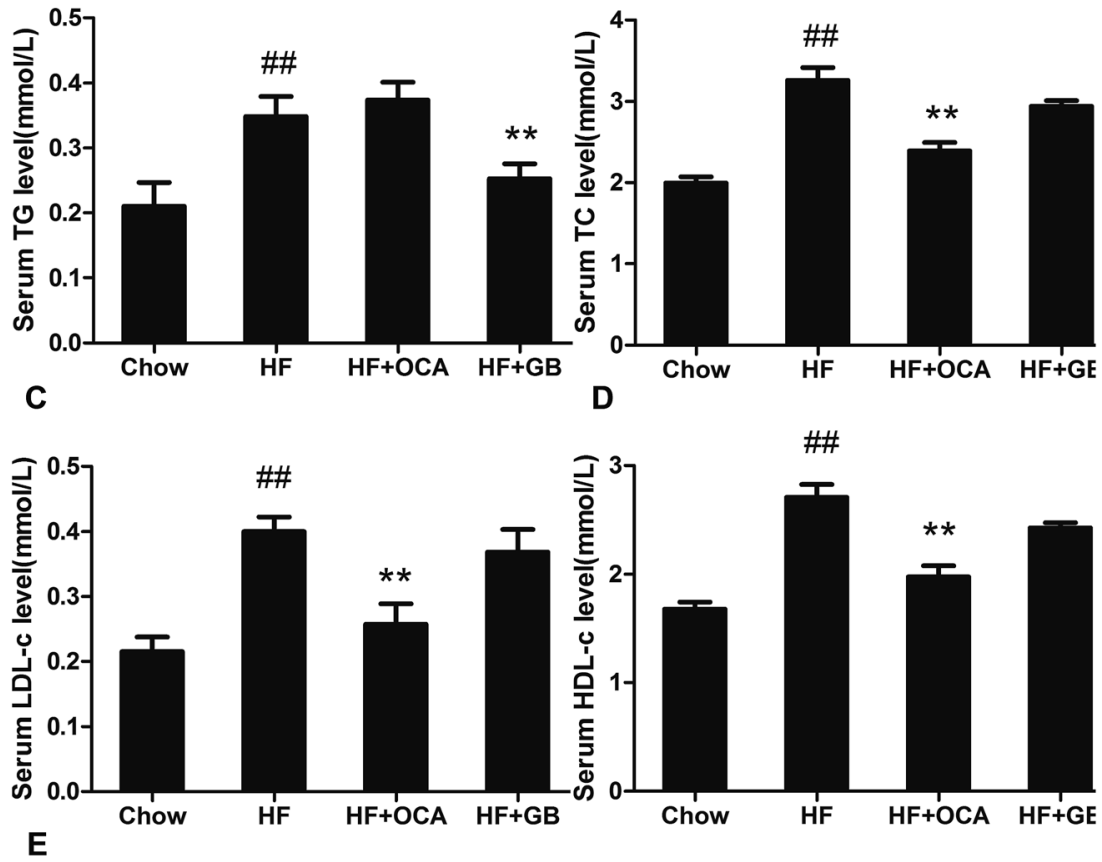

D

\#\#

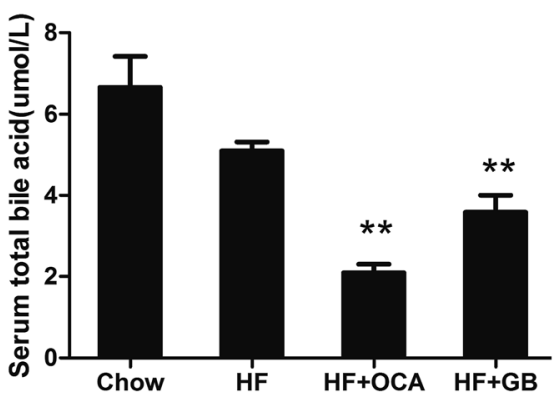

Fig. 3 Effects of ginkgolide B on serum lipid profile and bile acid contents in DIO mice. (A) Serum triglyceride (TG) levels. (B) Serum total cholesterol (TC) levels. (C) Serum low density lipoprotein cholesterol (LDL-C) levels. (D) Serum high density lipoprotein cholesterol (HDL-C). (E) Serum total bile acid. The data are shown as means $\pm \operatorname{SEM}(n=8)$. \#P<0.05, \#\#P<0.01, compared to the chow group; $* P<0.05, * * P<0.01$ compared to the HF group. 
a normal liver structure on regular chow diet-fed mice, whereas showed microvesicular and macrovesicular steatosis on the liver of DIO mice (Fig. 4A). In contrast, both GB and OCA treatment efficiently reversed the tissue structure and improved hepatic steatosis in the mice (Fig. 4A). Similarly, oil red O staining confirmed there was less lipid in the liver of chow-diet mice, but a massive lipid accumulation in liver of DIO mice. GB and OCA treatment notably attenuated the lipid accumulation in the liver of DIO mice (Fig. 4B). However, GB failed to reduce the serum ALT and AST levels in high fat-diet mice (Fig. 4C and D). Glutathione (GSH) is an important index of liver diseases, which can prevent damage from peroxides and lipid peroxides caused by oxidative stress. ${ }^{26}$ Our study showed GSH levels were remarkable lower in the liver of DIO mice when compared to that in chow diet-fed mice (Fig. 4E). GB significantly elevated GSH level in the live of DIO mice. Taken together, the data indicate that GB may improve hepatic steatosis.

\subsection{Ginkgolide B regulates the expression of PXR downstream genes}

To confirm the activation of PXR by GB, we analyzed the expression of PXR target genes in the liver and intestine of DIO mice. The results showed that GB significantly induced the mRNA expression of CYP3A11 and MRP3 in the liver tissues, whereas there was no difference expression of MRP2, MDR1 and CYP7A1 (Fig. 5A). Moreover, the expression of sterol regulatory element-binding protein (SREBP)-1, stearoyl-CoA desaturase (SCD)-1, acetyl-CoA carboxylase (ACC) and fatty acid synthase (FAS) was remarkably inhibited in the liver of GB treated mice (Fig. 5B). However, no obvious difference was observed in the expression of MCP-1, IL-1 $\beta$ and TNF- $\alpha$ in liver between the two groups (Fig. 5C). In addition, GB altered the expression of CYP3A11 in the intestine, while the expression of MDR1, IL-6, TNF- $\alpha$ and FGF15 was no significant difference (Fig. 5D).

\section{A}
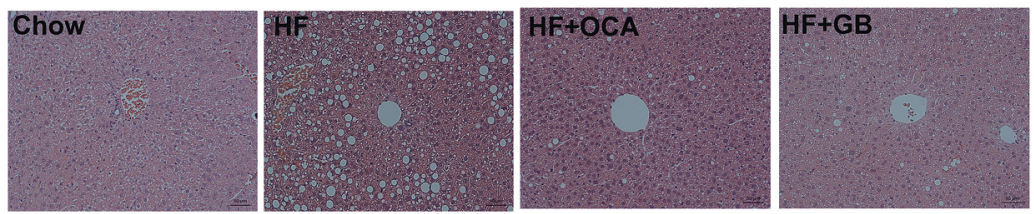

B

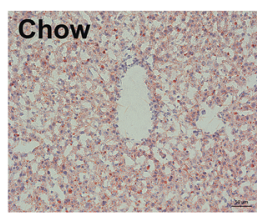

$\mathrm{C}$

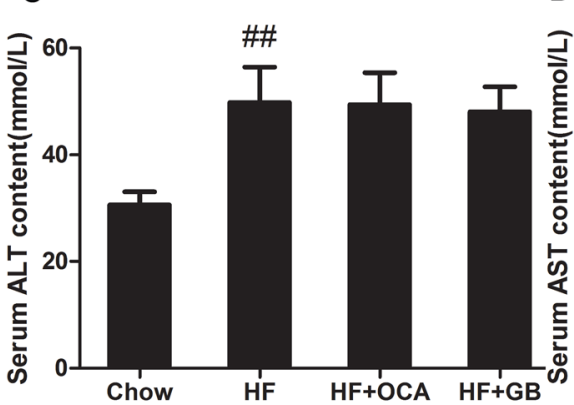

E

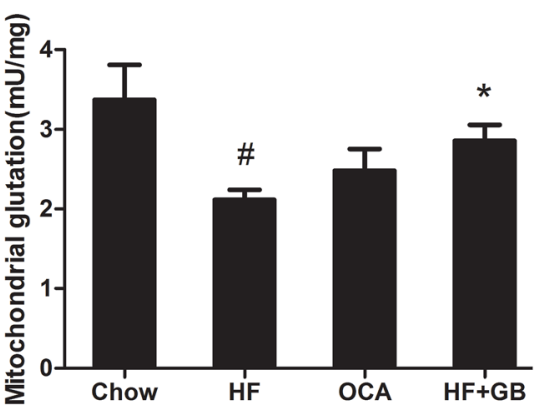

D
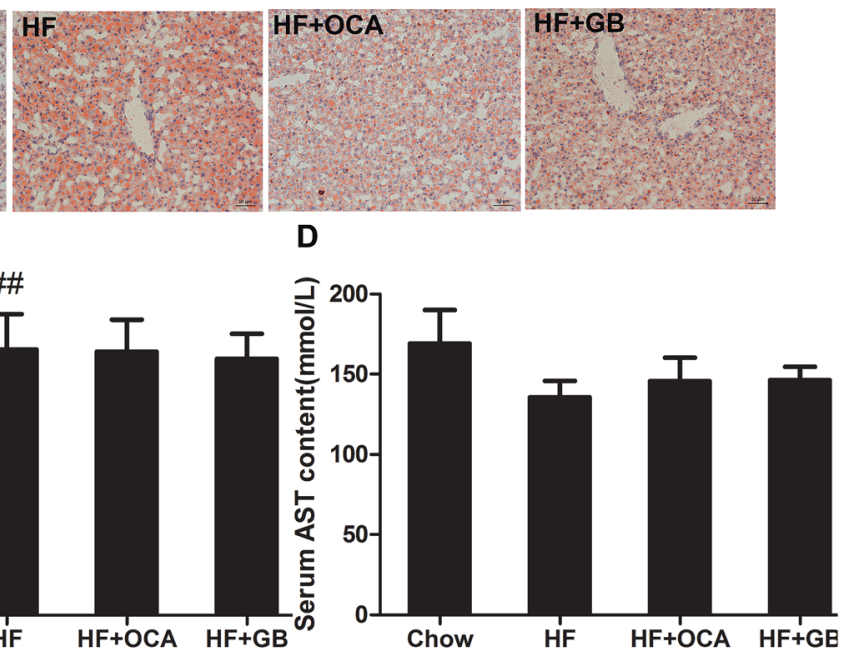

Chow HF HF+OCA HF+GE

Fig. 4 Ginkgolide B ameliorates hepatic steatosis in DIO mice. (A) H\&E staining of liver sections ( $\times 200)$. (B) Oil red O staining of liver sections

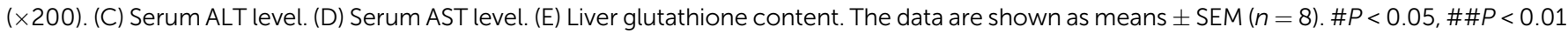
compared to the chow group; $* P<0.05, * * P<0.01$ compared to the HF group. 
A

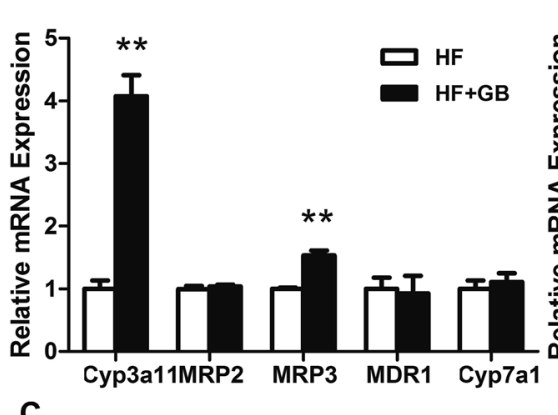

C

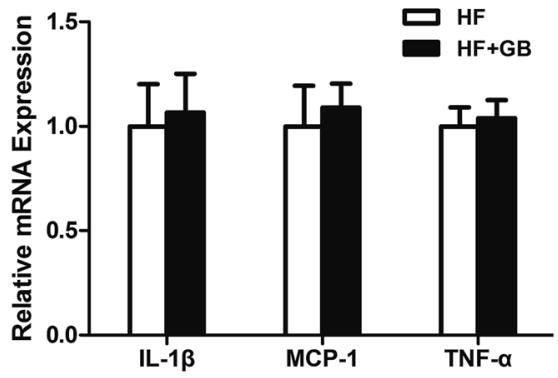

B

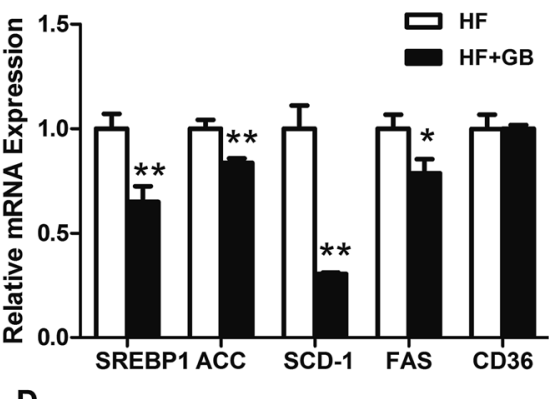

D

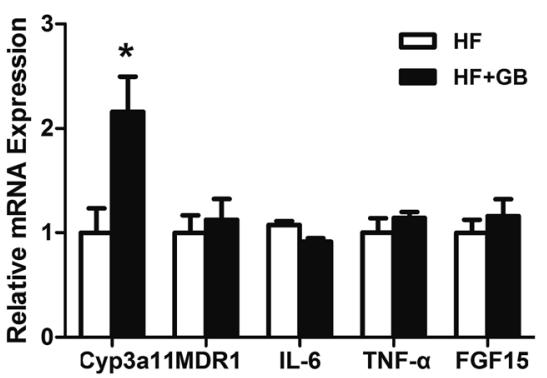

Fig. 5 Ginkgolide B regulates the gene expression in the liver and intestine of DIO mice. (A-C) The relative expression levels of genes in the liver. (D) The relative expression levels of genes in the intestine. For real-time PCR, the results were repeated in at least 3 independent experiments. $\beta$ Actin was used as an internal control for normalizing the mRNA level. The data are shown as means $\pm \operatorname{SEM}(n=3)$. $* P<0.05, * * P<0.01$ compared to the HF group.

\section{Discussion}

In this study, we provided the evidence that GB is a PXR agonist and could alleviate metabolic disorders in high-fat diet mice. Our results clearly showed that GB treatment blocked the body weight gain and improved hypertriglyceridemia in the DIO mice. Histologic evidence showed that GB attenuated hepatic fat droplets and improved liver morphology in DIO mice, suggesting that GB can be used to alleviate hepatic steatosis.

GB is an active component of Ginkgo biloba. It is reported that GB activated hPXR (human PXR) and rPXR (rat PXR), bound to the ligand binding domain of hPXR, recruited steroid receptor coactivator- 1 to hPXR and increased hPXR target gene (CYP3A4) expression. ${ }^{27,28}$ However, another study showed that GB did not increase hPXR and rPXR transactivity, ${ }^{29}$ and a timeresolved fluorescence resonance energy transfer competitive binding assay showed it is not bound to hPXR ligand-binding domain. ${ }^{29}$ In the present study, we observed that GB activated human PXR (hPXR) in a dose-depend manner by cell-based reporter assay. This result is similar to a previous report that showed GB activated human PXR. ${ }^{28}$

It is impressive that the previous studies have revealed the Ginkgo biloba extract could improve metabolic disorders in obese mice through targeting different receptors. ${ }^{17,30}$ However, we found GB only enhanced hPXR transactivity, but not $\operatorname{PPAR} \alpha$, PPAR $\beta$, PPAR $\gamma, L X R \alpha, L X R \beta$ and FXR transactivities. Herein, we suspected GB may regular metabolic through PXR pathway.

It has been reported that the activation of PXR may increase hepatic fatty acid and lipogenise and decrease $\beta$-oxidation. ${ }^{31,32}$
However, recent studies have shown that PXR agonists reduce the levels of lipids and glucose in diabetes models, ${ }^{33}$ and attenuate hepatic lipogenesis and gluconeogenesis in high-fat diet AKR/J mice. ${ }^{7}$ In addition, the ablation of PXR has been shown to increase hepatic steatosis, ${ }^{34}$ suggested a complex functions of PXR in the liver. Here, we found that GB prevented development of high-fat diet induced obesity, relieved obesityrelated hepatic lipid accumulation, elevated glutathione level in live tissues of DIO mice. Thus, this beneficial effect might be obtained by the activation of PXR signaling.

PXR is bile acid sensor, due to its induction of the gene expression of metabolizing enzymes CYP3A, UGT1A1, the uptake transporter OATP2 and the output transporter MRP3 leading to the clearance of bile acids from the liver. ${ }^{35} \mathrm{We}$ found that GB treatment reduced serum total bile acid (TBA) in DIO mice. And GB significantly up-regulated CYP3A11 and MRP3 mRNA expression in the liver of DIO mice, as well as the expression of CYP3A11 in small intestine, but not MRP2, MDR1 and CYP7A1. The data indicated that GB potentially involved in bile acid homeostasis via the activation of PXR.

In general, weight gain is deemed to a consequence of the increase in adipocyte mass and numbers caused by excess calories, ${ }^{36}$ while the specific mechanisms by which GB protects against weight gain is yet to be defined. There are several likely possibilities based on evidence available from our studies. We considered whether GB reduced the food intake. However, we did not observe the difference of food intake amount between the HF group and GB treatment group. We did not observed that the inhibition of food intake by GB. However, GB treatment increased the fecal TG contents. In previous studies, it has been 
reported that activation of PXR may remove excess lipid in the intestine. ${ }^{37}$ CYP3A11, the mouse ortholog of human CYP3A4, has been implicated in intestinal lipid metabolism. ${ }^{38}$ Thus, our data suggest that inhibition of TG absorption may be a mechanism for weight reducing of GB.

PXR plays important roles in regulating lipid homeostasis, though there was contradictory view that PXR agonists promote or inhibit adipogenesis. ${ }^{39}$ PXR activation by PCN reduced expression of SREBP-1, FAS, ACC. ${ }^{7}$ Similarly, our study showed that administration of GB displayed a marked reduction of de novo lipogenesis with inhibited expression of SREBP-1, and primary lipogenic enzymes FAS, ACC and SCD-1 in the liver tissues, which consistent with the effect of PCN. Our result further proved that GB could improve metabolic disorders through the regulation of PXR signaling.

PXR activation is generally anti-inflammatory across many tissues. The human PXR agonist rifampicin suppressed NF-kB proinflammatory target genes cyclooxygenase (COX)-2 and TNF- $\alpha{ }^{40}$ However, our study shown that GB displayed no change of the expression of MCP-1, IL-1 $\beta$ and TNF- $\alpha$ in the liver of DIO mice, although Ginkgo biloba extract attenuates inflammation in retroperitoneal adipose tissue depot of obese rats. ${ }^{30}$ This difference may be caused by the animal model. In our study, the liver of DIO mice did not show significant inflammation response.

In summary, we demonstrated that GB may have beneficial effects on metabolic disorders of DIO mice, possibly through the activation of PXR, resulting in blocking the body weight gain, attenuating hypertriglyceridemia and hepatic steatosis, and improving bile acid homeostasis in DIO mice. The mechanistic study showed that GB improved metabolic disorders possibly through the activation of PXR. Additional work is needed to illustrate the precise mechanisms of GB modulating lipid homeostasis. Our results indicate that GB may be a specific activator for human PXR, and could be used as a potential treatment for metabolic disorders.

\section{References}

1 Y. Y. Ji and S. S. Kim, Nutrients, 2016, 8, 173.

2 P. Zimmet, D. Magliano, Y. Matsuzawa, G. Alberti and J. Shaw, J. Atheroscler. Thromb., 2005, 12, 295-300.

3 J. P. Ong and Z. M. Younossi, Clin. Liver Dis., 2007, 11, 1-16. 4 J. M. Lehmann, D. D. Mckee, M. A. Watson, T. M. Willson, J. T. Moore and S. A. Kliewer, J. Clin. Invest., 1998, 102, 1016-1023.

5 C. Zhou, S. Verma and B. Blumberg, Nucl. Recept. Signaling, 2009, 7, e001.

6 S. Kodama, R. Moore, Y. Yamamoto and M. Negishi, Biochem. J., 2007, 407, 373-381.

7 Y. Ma and D. Liu, PLoS One, 2012, 7, e38734.

8 J. Zhou, M. Febbraio, T. Wada, Y. Zhai, R. Kuruba, J. He, J. H. Lee, S. Khadem, S. Ren and S. Li, Gastroenterology, 2008, 134, 556-567.

9 A. Bitter, P. Rümmele, K. Klein, B. A. Kandel, J. K. Rieger, A. K. Nüssler, U. M. Zanger, M. Trauner, M. Schwab and O. Burk, Arch. Toxicol., 2015, 89, 2089-2103.
10 K. M. Maclennan, C. L. Darlington and P. F. Smith, Prog. Neurobiol., 2002, 67, 235-257.

11 P. Braquet, Adv. Prostaglandin, Thromboxane, Leukotriene Res., 1986, 16, 179-198.

12 X. H. Liu, B. L. Eun, F. S. Silverstein and J. D. Barks, Pediatr. Res., 1996, 40, 797-803.

13 E. Koch, Phytomedicine, 2005, 12, 10-16.

14 J. H. Gu, J. B. Ge, M. Li, F. Wu, W. Zhang and Z. H. Qin, Eur. J. Pharm. Sci., 2012, 47, 652-660.

15 L. Sun, Z. He, J. Ke, S. Li, X. Wu, L. Lian, X. He, X. He, J. Hu and Y. Zou, Int. J. Clin. Exp. Pathol., 2015, 8, 432-440.

16 G. Allais, G. D'Andrea, M. Maggio and C. Benedetto, Neurol. Sci., 2013, 34, 161-163.

17 X. Gu, Z. Xie, Q. Wang, G. Liu, Y. Qu, L. Zhang, J. Pan, G. Zhao and Q. Zhang, FEBS J., 2009, 276, 1450-1458.

18 Y. Kanno, T. Yatsu, N. Yamashita, S. Zhao, W. Li, M. Imai, M. Kashima, Y. Inouye, K. Nemoto and K. Koike, Phytomedicine, 2017, 26, 22-27.

19 W. Dou, J. Zhang, H. Li, S. Kortagere, K. Sun, L. Ding, G. Ren, Z. Wang and S. Mani, J. Nutr. Biochem., 2014, 25, 923-933.

20 B. Mlinar, J. Marc and M. Pfeifer, Biochem. Med., 2006, 16, 824.

21 B. A. Neuschwandertetri, R. Loomba, A. J. Sanyal, J. E. Lavine, M. L. V. Natta, M. F. Abdelmalek, N. Chalasani, S. Dasarathy, A. M. Diehl and B. Hameed, Lancet, 2014, 385, 956-965.

22 A. Yokota, S. Fukiya, K. B. Islam, T. Ooka, Y. Ogura, T. Hayashi, M. Hagio and S. Ishizuka, Gut Microbes, 2012, 3, 455.

23 J. F. Pierre, K. B. Martinez, H. Ye, A. Nadimpalli, T. C. Morton, J. Yang, Q. Wang, N. Patno, E. B. Chang and D. P. Yin, Am. J. Physiol.: Gastrointest. Liver Physiol., 2016, 311, 286-304.

24 A. H. Ali and K. D. Lindor, Expert Opin. Pharmacother., 2016, 17, 1809-1815.

25 A. S. Cikim, E. Topal, M. Harputluoglu, L. Keskin, Z. Zengin, K. Cikim, R. Ozdemir, M. Aladag and S. Yologlu, J. Endocrinol. Invest., 2007, 30, 459-464.

26 J. C. Fernándezcheca, N. Kaplowitz, C. Garcíaruiz, A. Colell, M. Miranda, M. Marí, E. Ardite and A. Morales, Am. J. Physiol., 1997, 273, 7-17.

27 A. J. Lau, G. Yang, C. W. Yap and T. K. Chang, Drug Metab. Dispos., 2012, 40, 1113-1121.

28 A. H. Tolson, Pharm. Res., 2009, 26, 872-882.

29 A. J. Lau, G. Yang, G. Rajaraman, C. C. Baucom and T. K. Chang, J. Pharmacol. Exp. Ther., 2010, 335, 771-780.

30 B. K. Hirata, R. M. Banin, A. P. Dornellas, I. S. de Andrade, J. C. Zemdegs, L. C. Caperuto, L. M. Oyama, E. B. Ribeiro and M. M. Telles, Mediators Inflammation, 2015, 2015, 1-9.

31 J. Zhou, Y. Zhai, Y. Mu, H. Gong, H. Uppal, D. Toma, S. Ren, R. M. Evans and W. Xie, J. Biol. Chem., 2006, 281, 1501315020.

32 K. Spruiell, R. M. Richardson, J. M. Cullen, E. M. Awumey, F. J. Gonzalez and M. A. Gyamfi, J. Biol. Chem., 2014, 289, 3244-3261.

33 W. X. Jie Gao, Drug Metab. Dispos., 2010, 38, 2091-2095. 
34 K. Spruiell, R. M. Richardson, J. M. Cullen, E. M. Awumey, F. J. Gonzalez and M. A. Gyamfi, J. Biol. Chem., 2014, 289, 3244-3261.

35 J. L. Staudinger, B. Goodwin, S. A. Jones, D. Hawkins-Brown, K. I. MacKlenzie, A. LaTour, Y. Liu, C. D. Klaasseni, K. K. Brown, J. Reinhard, T. M. Willson, B. H. Koller and S. A. Kliewer, Proc. Natl. Acad. Sci. U. S. A., 2001, 98, 33693374.

36 J. S. Flier, Cell, 2004, 116, 337-350.
37 T. Li, W. Chen and J. Y. Chiang, J. Lipid Res., 2007, 48, 373384.

38 B. Morón, A. K. Verma, P. Das, J. Taavela, L. Dafik, T. R. Diraimondo, M. A. Albertelli, T. Kraemer, M. Mäki and C. Khosla, Am. J. Gastroenterol., 2013, 108, 1344-1351.

39 M. Wagner, G. Zollner and M. Trauner, Hepatology, 2011, 53, 1023-1034.

40 C. Zhou, M. M. Tabb, E. L. Nelson, F. Grün, S. Verma, A. Sadatrafiei, M. Lin, S. Mallick, B. M. Forman and K. E. Thummel, J. Clin. Invest., 2006, 116, 2280-2289. 